\title{
Mechanical Effects of Canes on Postural Control: Beyond Perceptual Information
}

\author{
Marta Russo ( $\nabla$ m.russo@hsantalucia.it ) \\ Jongwoo Lee \\ MIT: Massachusetts Institute of Technology \\ Neville Hogan \\ Massachusetts Institute of Technology \\ Dagmar Sternad \\ Northeastern University
}

Fondazione PTV Policlinico Tor Vergata https://orcid.org/0000-0003-1020-7542

Research

Keywords: postural control, balance, haptic support, mechanical support, stability

Posted Date: November 15th, 2021

DOI: https://doi.org/10.21203/rs.3.rs-1061413/v1

License: (c) (1) This work is licensed under a Creative Commons Attribution 4.0 International License.

Read Full License 
1

3

4

5

6 1: m.russo@hsantalucia.it, Department of Biology, Northeastern University, Boston, MA, USA \&

7 Department of Neurology, Tor Vergata Polyclinc, Rome, Italy

8 2: jw127@mit.edu, Department of Mechanical Engineering, Massachusetts Institute of

9 Technology, Cambridge, MA, USA

10 3: neville@mit.edu, Department of Mechanical Engineering \& Department of Brain and Cognitive

11 Sciences, Massachusetts Institute of Technology, Cambridge, MA, USA

12 4: d.sternad@northeastern.edu, Department of Biology, Electrical and Computer Engineering, and

13 Physics, Northeastern University, Boston, MA, USA

14

$15{ }^{*}$ Corresponding Author:

16

Marta Russo m.russo@hsantalucia.it,Department of Neurology, Tor Vergata Polyclinc, Rome, Italy
Formatted: Font color: Text 1

Formatted: Font color: Text 1

Formatted: Font color: Text 1 
2 Background: Numerous studies showed that postural balance improves through light touch on a

3 stable surface highlighting the importance of haptic information, seemingly downplaying the 4 mechanical contributions of the support. The present study examined the mechanical effects of 5 canes for assisting balance in healthy individuals challenged by standing on a beam.

6 Methods: Sixteen participants supported themselves with two canes, one in each hand, and applied 7 minimal, preferred, or maximum force onto the canes. They positioned the canes in the frontal 8 plane or in a tripod configuration.

9 Results: Results showed that canes significantly reduced the variability of the center of pressure and 10 center of mass to the same level as when standing on the ground. In the preferred condition, 11 participants exploited the altered mechanics by resting their arms on the canes and, in the tripod 12 configuration, allowing for larger CoP motions in the task-irrelevant dimension. Increasing the 13 exerted force beyond the preferred level yielded no further benefits, in fact had a destabilizing 14 effect on the canes: the displacement of the hand on the cane handle increased with the force.

15 Conclusions: Despite the challenge of a statically unstable system, these results show that, in 16 addition to augmenting perceptual information, using canes can provide mechanical benefits and 17 challenges. First, the controller minimizes effort channeling noise in the task-irrelevant dimensions 18 and, second, resting the arms on the canes but avoiding large forces that would have destabilizing 19 effects. However, if maximal force is applied to the canes, the instability of the support needs to be 20 counteracted, possibly by arm and shoulder stiffness.

22 Keywords: postural control, balance, haptic support, mechanical support, stability 
2 In the last century many neuroscientists focused their attention on the role of sensory information

3 for postural control. Not surprisingly, visual input together with vestibular and proprioceptive 4 information provides strong support for maintain balance $(19,20)$. Less intuitive is the role of haptic

5 information, such as through touching a surface or holding the hand of another person. A seminal 6 study has shown that light touch of the fingertip on an earth-fixed surface significantly reduced CoP 7 motion (11). Increasing the amount of force applied on the surface had minimal effect on the CoP 8 motion. However, most subsequent studies on light touch tested participants' ability to balance 9 using earth-fixed supports that were stationary. The situation is different when relying on a 10 supporting device. Typically, a cane or holding the hand of another person does not present a stable 11 stationary support. Yet, as seen in the frequent use of sticks or poles in hiking, such nonstationary 12 devices or another person do appear to provide stability (8).

13 A small number of studies have attempted to understand the support provided by unstable or 14 unreliable support during standing or walking. Blind individuals who only have haptic information 15 from the 'white cane' to rely on reported that force levels significantly below those that provided 16 physical support presented useful stabilization (10). Jeka and collaborators confirmed that touch 17 with a cane, only indirect contact of a surface through a hand-held stick, reduced postural sway. In 18 the same vein, a recent study examined bimanual support gathered via two ropes held in each hand 19 and anchored to the ground on the other extremity (18). The researchers compared CoP motion in 20 this scenario with a bimanual light touch obtained via two earth-fixed supports. The latter proved 21 superior in reducing CoP motion and variability, albeit both support conditions improved postural 22 stance with respect to standing with the hands free. In addition, it has been shown that light touch 23 even of a curtain, an unreliable reference, benefitted postural stability (25). In a similar study, the 24 authors confirmed that unrealiable touch reference affects CoP motion, albeit they observed an 
1 increase in CoP area after the perturbation of the reference. They speculated that an unrealible

2 touch reference might engage a reweighting mechanism of tactile perceptual cues (3).

3 The role of light touch becomes even more effective when standing on an unstable surface such as 4 a foam pad or a beam (15-17). When standing on a beam elevated above ground, earth-fixed light 5 touch reduced CoP motion both with and without visual input (15). The same study also showed 6 that bimanual touch improved postural balance, especially when standing on a higher beam where

7 fear of falling became an issue. This wide range of studies consolidated the role of perceptual 8 information provided through light touch.

9 In this solid demonstration of the perceptual support through light touch, little attention was paid 10 to the also present mechanical aspects. The force levels that have been investigated in previous 11 studies on light touch typically ranged from $<1 \mathrm{~N}$ to about $10 \mathrm{~N}$. At such low force levels, mechanical 12 benefits were indeed likely to be subordinate. However, support also affords leaning on it, as 13 frequently done when walking with a cane. Further, when standing on an challenging support 14 surface, such as a narrow beam or uneven ground, mechanical assistance from a cane likely plays a 15 much greater role. Hence, the aim of the present study was to investigate the mechanical 16 contribution to postural balance of support through hand-held canes. To challenge postural 17 stability, participants were asked to stand on a narrow beam on the ground in tandem stance. In 18 that configuration, the base of support was strictly limited to the beam dimensions, in particular to 19 its width. To help participants to maintain balance, they held two canes, one in each hand and 20 placed on the ground. For reference, we also included two control conditions: standing on the beam 21 without canes, and standing on the ground, also in tandem stance.

22 To establish a baseline, we first evaluated the effect of the beam on postural stability, comparing 23 the variability of the center of mass and center of pressure while standing on the ground with 
1 standing on the beam. Further, we assessed the contribution of cane support compared to standing

2 on the beam without canes.

3 To examine mechanical contributions to postural stability from cane support, increasing force levels 4 were investigated. Participants were asked to apply minimal, preferred, and maximum levels of 5 force on the two canes, where the maximal force condition significantly exceeded the force range 6 studied in earlier work. We reasoned that greater forces applied on the canes will have significant 7 mechanical effects on postural stability, and expected that the variability of the center of pressure 8 will be reduced (Hypothesis 1 ).

9 Nevertheless, these canes presented an additional challenge: a cane is an inverted pendulum that 10 is inherently unstable. When applying higher forces onto the canes, noise may increase 11 proportionally with force as it is regarded as signal-dependent (13). In addition, it was previously 12 shown that exerting a compression force on a mechanical rod induces mechanical instability, such 13 that the cane may be easily pushed over with any small perturbation $(22,23)$. Hence, higher forces 14 onto the canes can have the opposite effect and destabilize posture. Based on these two effects, 15 we expected that the canes will show more variability at higher forces (Hypothesis 2).

16 From a mechanical perspective the configuration of the canes can have a significant effect. To 17 examine how the configuration of cane placement affects postural stability, participants were 18 instructed to place the canes in two different arrangements. In the first 'planar' condition, 19 participants held the canes symmetrically with their arms extended to their sides. Standing on a 20 beam in tandem stance has a large destabilizing effect in the medio-lateral direction. Therefore, by 21 placing the canes in the frontal plane, participants can directly exert angular momentum and 22 compensate the medio-lateral component of the center of mass and center of pressure motion (4). 23 In the second 'tripod' condition, canes were placed in front of the body, with both arms at $45^{\circ}$ angle 
1 with the frontal plane. Due to the increased base of support, we reasoned that the tripod condition

2 will significantly improve postural balance. While it will contribute to the medio-lateral direction, it

3 will also allow more room for motion in the antero-posterior direction, without destabilizing the

4 posture. As there is invariably noise-induced variability in the center of pressure, this configuration

5 could indeed channel noise in this task-irrelevant dimension, i.e., variability in the AP direction will 6 be enhanced (Hypothesis 3).

7 To sensitively evaluate the intricate mechanical effects, ground reaction forces were measured both

8 at the feet and at the canes. The beam was placed on a force plate and 6-DOF force sensors

9 measured forces applied on the canes. The latter allowed separate quantification of the center of 10 pressure at the feet and over the total support area. Based on 3D kinematic recordings, we assessed 11 the motion of the hands on the canes and also estimated the center of mass motion.

\section{Methods}

\section{Participants}

1416 participants (7 females, 9 males, between 19 and 36 years) with no history of neurological 15 conditions and normal or corrected-to-normal vision took part in the experiments upon signing the 16 informed consent form. The study was approved by the Institutional Review Board of Northeastern 17 University.

\section{Experimental Apparatus}

19 Participants stood on a narrow wooden beam (width $3.65 \mathrm{~cm}$, height $7.62 \mathrm{~cm}$ ) that was placed on 20 the floor on top of a force plate (AMTI, Watertown, MA, Figure 1). They held two aluminum canes, 21 one in each hand, to support themselves (length $117 \mathrm{~cm}$, mass $680 \mathrm{~g}$ ). The two canes were 22 instrumented with a 6-DOF load cell at the bottom of each cane to measure the forces applied to 
1 the canes (MCW-500 Walker Sensors, AMTI Watertown, MA). All force data were recorded at 500

$2 \mathrm{~Hz}$ sampling rate. To record the participants' movements in 3D space, whole-body kinematics were

3 recorded by 12 optoelectronic cameras at a sampling rate of $100 \mathrm{~Hz}$ (Qualisys $A B$, Göteborg,

4 Sweden). Each participant was equipped with a standard biomechanical set of 43 reflective markers,

5 following the C-Motion Plug-In Gait marker set. To track the orientation of the canes in 3D space, 4 6 additional markers were attached to each cane.

\section{$8 \quad$ Experimental Protocol}

9 Participants were asked to stand barefoot in tandem stance on the narrow beam without stepping 10 on the ground. They could choose which foot was at the front of their stance and they kept the 11 same foot in front in all trials. For all experimental conditions, participants supported themselves 12 with two canes, one held in each hand, their arms comfortably extended. Participants were asked 13 to apply one of three levels of force on the canes: minimum (Min), i.e., as little as they could, 14 preferred (Pref), i.e., as much as they liked, maximum (Max), i.e., as much as possible. They 15 performed the same three force conditions in two arm configurations: their arms extended out 16 horizontally in the frontal plane (Planar), and stretched out forward forming approximately a $45^{\circ}$ 17 angle at the shoulder with the frontal plane (Tripod). The Planar configuration limited the base of 18 support to a line, while the Tripod significantly enlarge the base to a triangular area. In addition, 19 standing on a narrow beam elicited instability around the vertical, but mainly confined to the frontal 20 plane. Therefore, we chose the Planar configuration to counter such effect directly in the frontal 21 plane. The rationale for choosing straight arms was twofold: first, this simple joint configuration 22 minimized differences across individuals and, second, it eliminated additional stiffness of the elbow 23 and the wrist joints acting at the hand/cane junction. In one additional control condition participants 24 stood on the beam without the cane support (On Beam). In this difficult condition they were allowed 
1 to move their arms freely to help maintain balance on the beam. We further recorded a baseline

2 condition in which participants stood on the ground in the same tandem stance without holding 3 canes (Off Beam).

4 Each combination of arm configurations and force on the canes was repeated three times,

5 performed in blocks. Each block presented all experimental conditions once (3 force levels: Min vs.

6 Pref vs. Max in 2 arm configurations: Planar vs. Tripod). Each trial lasted $30 \mathrm{~s}$; the entire recording

7 session lasted approximately one hour, including placing the markers on the body.

\section{$8 \quad$ Data Preprocessing}

9 All analyses were carried out with custom software written in Matlab (The Mathworks Inc., Natick, 10 MA). All kinematic and kinetic data were filtered with a zero-lag $3^{\text {rd }}$-order low-pass Butterworth 11 filter at $10 \mathrm{~Hz}$ (functions: butter, filtfilt). In order to exclude any transient or fatigue effects, data 12 from the first $10 \%$ and last $10 \%$ of each trial were excluded from the analysis. The weight of the 13 cane was subtracted from the vertical component of the force measured at the canes, to estimate 14 the effective force applied by participants. In trials with cane support, participants did not step off 15 the beam during the trial, hence no further action was necessary. In the control condition where 16 participants stood on the beam without cane support, they occasionally lost balance and stepped 17 off the beam. These trials were excluded from the analysis.

18 As the feet of the participant were not in direct contact with the force plate but with the beam, the 19 the center of pressure recorded by the force plate (Ground-CoP) was different from that resultant 20 from the feet-beam interaction (Beam-CoP). The discrepancy was evaluated by the following 21 equations,

$$
\text { Beam-CoP }=\text { Ground-CoP } \mathrm{P}_{\mathrm{x}}+h \frac{F_{x}^{g}}{F_{z}^{g}}
$$




$$
\text { Beam-CoP } y=\text { Ground-CoP } y+h \frac{F_{y}^{g}}{F_{z}^{g}}
$$

2 where $\mathrm{h}$ is the height of the beam and $F^{g}=\left[\mathrm{F}_{\mathrm{x}}^{\mathrm{g}}, \mathrm{F}_{\mathrm{y}}^{\mathrm{g}}, \mathrm{F}_{\mathrm{z}}^{\mathrm{g}}\right]$ is the ground reaction forces recorded by

3 the force plate. The $\mathrm{x}$-axis corresponded to the ML direction, the $\mathrm{y}$-axis to the AP direction, and the

$4 \quad z$-axis to the vertical direction, as illustrated in Fig. 1.

Formatted: Font color: Text 1

Formatted: Font color: Text 1

Formatted: Font color: Text 1

Formatted: Font color: Text 1

5 As $F_{z}{ }^{g}>>F_{x, y}{ }^{g}$, the additional term on the right side of equation (1) and (2)

6 Error! Reference source not found.was negligible. Thus, in the following only the Ground-CoP was

7 considered. For the sake of clarity, the CoP on the ground was referred to as the Feet-CoP.

8 When two canes touched the floor, the participant had three regions of contact with the ground:

9 the feet on the beam, and the tips of the two canes. The feet were on the beam, which was located 10 on the force platform, thus measuring the ground reaction force and the center of pressure.

11 Information about the force applied on the canes was provided by the load cells at the tip of the 12 canes. The center of pressure of each cane was computed by the ratio between the moments, $m_{x}$ 13 and $m_{y}$, and the forces, $f_{z}$, measured by the load cells.

$$
\operatorname{cop}_{x}=\frac{m_{y}}{f_{z}}
$$

$$
\operatorname{cop}_{y}=\frac{m_{x}}{f_{z}}
$$

16 The spatial positions of the tips of the canes were determined from the markers attached to the 17 canes. With all variables in the laboratory coordinate frame, the total center of pressure (Total-CoP) 18 was computed as the ratio of the total moments, $M_{x, y}$, and the total force, $F_{z}$. The total moments 19 were defined as the sum of the product of the vertical force at each point of contact with the 
1 respective moment arm. The moment arm at each point was computed as the sum of the center of

2 pressure with the relative position $a=\left[\mathrm{a}_{\mathrm{x}}, \mathrm{a}_{\mathrm{y}}, 0\right]$, which in turn is the vector from the origin of the

3 coordinate frame to the point of contact. As it was desirable to compute the CoP in the medio-

4 lateral $(\mathrm{ML})$ and antero-posterior $(\mathrm{AP})$ directions, the total moments in the AP and ML directions

5 were determined, respectively, as shown in equations 5 and 6

$6 \quad(5)$

$$
M_{x}=\sum_{i=1}^{3}\left(a_{y}^{i}+c o p_{y}^{i}\right) f_{z}^{i}
$$

$7 \quad(6)$

$$
M_{y}=\sum_{i=1}^{3}\left(a_{x}^{i}+\operatorname{cop}_{x}^{i}\right) f_{z}^{i}
$$

8 The index $i$ indicates the current point of contact ( $\mathrm{i}=1$ : feet, $\mathrm{i}=2$ : left cane, $\mathrm{i}=3$ : right cane). Following 9 the rule applied previously, the total CoP was determined as

10

$$
\text { Total-CoP }=\frac{\mathrm{M}_{\mathrm{y}}}{\mathrm{F}_{\mathrm{z}}}
$$

$11(8)$

$$
\text { Total- } \operatorname{CoP}_{\mathrm{y}}=\frac{\mathrm{M}_{\mathrm{x}}}{\mathrm{F}_{\mathrm{z}}}
$$

12 where $F_{z}=\sum_{i=1}^{3} f_{z}^{i}$.

13 For each participant a kinematic model of 15 rigid body segments (head, trunk, pelvis, left and right 14 upper arms, forearms, hands, thighs, shanks and feet) was fit to the kinematic data using C-Motion 15 Visual3D (Germantown, MD). The whole-body center of mass (CoM) was computed in Visual3D.

\section{Dependent Measures}

17 To obtain a metric for postural sway, the fluctuations of the CoP were summarized by the standard 18 deviations of the CoP in two orthogonal directions, the AP and ML directions. These two directions
Formatted: Font color: Text 1

Formatted: Font color: Text 1

Formatted: Font color: Text 1

Formatted: Font color: Text 1

Formatted: Font color: Text 1

Formatted: Font color: Text 1 
1 were calculated separately, because of the anisotropic constraints of the beam, i.e., the base of

2 support on the beam was larger in the AP direction than in ML. Another measure of postural sway

3 was defined as the area of the $95 \%$ tolerance ellipse. The same metrics were computed for both

4 Feet-CoP and Total-CoP. In addition, the fluctuations of the center of mass (CoM) were quantified

5 by the area of the $95 \%$ tolerance ellipse. This area was calculated in the horizontal (x-y) plane to

6 make it comparable to the areas of the CoPs. Note that movements in the vertical (z) direction were

7 negligible. To quantify movements of the hand at the tip of the cane, the path length of the hand

8 movement was calculated as the integral of the root mean squared sum of the derivatives of the $\mathrm{x}-$,

$9 \mathrm{y}$ - and z-components.

$$
\text { path length }=\int_{\text {start }}^{\text {end }} \sqrt{\left(\frac{d x}{d t}\right)^{2}+\left(\frac{d y}{d t}\right)^{2}+\left(\frac{d z}{d t}\right)^{2}} d t
$$

\section{Statistical Analysis}

13 A linear mixed model was used to evaluate the differences in the variability of the center of mass

14 and the center of pressure between the three levels of force applied to the canes and the two cane

15 configurations. The mixed model compared the experimental conditions (fixed effects), i.e., beam,

16 force and cane configuration conditions, which were consistent across participants, and accounted

17 for the effects of normally distributed variability between participants (random effects). In equation

18 (10) $Y$ is the latent response variable for each participant $i$ and each trial $j$, B is the beam condition

19 (On the Beam or On the Ground); $F$ is the force condition (three levels: Min, Pref, Max), $C$ is the arm

20 configuration (two levels: Planar and Tripod), $\beta$ are the fixed-effects coefficients, $S$ are the random-

21 effects coefficients.

$22 \quad(10)$

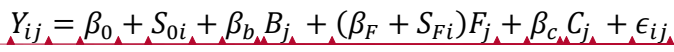

\begin{tabular}{|l|}
\hline Formatted: Font color: Text 1 \\
\hline Formatted: Font color: Text 1 \\
\hline Formatted: Font color: Text 1 \\
\hline Formatted: Font color: Text 1 \\
\hline Formatted: Font color: Text 1 \\
\hline Formatted: Font color: Text 1 \\
\hline Formatted: Font color: Text 1 \\
\hline Formatted: Font color: Text 1 \\
\hline Formatted: Font color: Text 1 \\
\hline Formatted: Font color: Text 1 \\
\hline Formatted: Font color: Text 1 \\
\hline Formatted: Font color: Text 1 \\
\hline Formatted: Font color: Text 1 \\
\hline Formatted: Font color: Text 1 \\
\hline Formatted: Font color: Text 1 \\
\hline Formatted: Font color: Text 1 \\
\hline
\end{tabular}


1 To better compare the force conditions in which participants were standing on the beam with the

2 canes on the ground, a second model (see equation 11) was tested on a subset of the data, excluding

3 trials in the control conditions.

$4 \quad(11)$

$$
Y_{i j_{\alpha}}=\beta_{0,}+S_{0 i}+\left(\beta_{F}+S_{F i}\right) F_{j_{\Delta}}+\beta_{c_{\mu}} C_{j_{\Delta}}+\epsilon_{i j_{\mathrm{A}}}
$$

5 Additional multiple comparisons were conducted across experimental conditions by pairwise t-tests

6 with Bonferroni correction.

7 All statistical analyses were carried out in R, with packages stats, Ime4 and ImerTest (21).

\section{Results}

9 This study examined the mechanical effect of cane support for maintaining standing balance.

10 Specifically, the experiment aimed to identify the mechanical effects of two canes on the control of 11 balance when standing on a narrow beam. Participants supported themselves by holding two canes 12 placed either on their side or in a tripod configuration (Figure 1). In the latter placement, the arms 13 formed a 45 deg angle and the canes were in front of the body, forming a triangle with the feet. 14 Participants were asked to exert three levels of force onto the canes: minimum (Min), preferred 15 (Pref), and maximum (Max). We measured the displacements of the center of pressure on the beam, 16 the forces on the canes, the body's center of mass, and the displacements of the hands at the cane. 17 To provide a baseline measure, both the center of pressure and the center of mass were quantified 18 when participants stood on the ground, in the same tandem foot position as on the beam. Another 19 reference measure was obtained when participants stood on the beam without cane support. The 20 overarching question of this study was how the different forces applied to the canes and two arms 21 configurations affected the mechanics and, hence, the control of postural balance.

\begin{tabular}{|l|}
\hline Formatted: Font color: Text 1 \\
\hline Formatted: Font color: Text 1 \\
\hline Formatted: Font color: Text 1 \\
\hline Formatted: Font color: Text 1 \\
\hline Formatted: Font color: Text 1 \\
\hline Formatted: Font color: Text 1 \\
\hline Formatted: Font color: Text 1 \\
\hline Formatted: Font color: Text 1 \\
\hline Formatted: Font color: Text 1 \\
\hline Formatted: Font color: Text 1 \\
\hline Formatted: Font color: Text 1 \\
\hline Formatted: Font color: Text 1 \\
\hline Formatted: Font color: Text 1 \\
\hline
\end{tabular}


1 Forces Applied on the Canes. The first test verified that participants indeed followed instructions

2 and applied different forces on the canes. Table 1 shows the summed vertical forces applied on the

3 two canes averaged over the duration of the trial. For the three force instructions and for the two

4 cane placements the applied forces ranged between 4 and $50 \mathrm{~N}$ for each cane. The linear mixed

5 model confirmed the difference between the three force instructions with a significant main effect

$6 \quad(\beta=10.1 \pm 2.6, p<0.001)$. All three force conditions were larger than those examined in previous

7 studies and the preferred force differed from both the maximum and the minimum forces. The two

8 cane configurations did not elicit different forces in the three force conditions $(\beta=-0.7 \pm 4.3, p=$

9 0.86).

\begin{tabular}{l|l|l|l}
\hline & MIN (M+/-SD) & PREF (M+/-SD) & MAX (M+/-SD) \\
\hline POSTURE & & & \\
\hline PRIPOD & $8.25+/-10.08 \mathrm{~N}$ & $33.21+/-11.97 \mathrm{~N}$ & $91.20+/-36.82 \mathrm{~N}$ \\
\hline & $9.64+/-12.15 \mathrm{~N}$ & $32.87+/-12.28 \mathrm{~N}$ & $85.60+/-31.71 \mathrm{~N}$ \\
\hline
\end{tabular}

Formatted: Font color: Text 1

Formatted: Font color: Text 1

10 Table 1: Sum of forces applied on the canes. Means and standard deviations across participants of

11 the sum of the forces applied on the two canes in the three force conditions and in the two postures.

12 Forces were averaged across the duration of the trial.

13 The preferred force applied on both canes was reliably around $33 \mathrm{~N}$ in both arm configurations,

14 corresponding to the weight of a $3.36 \mathrm{~kg}$ mass. Assuming the weight of one arm is $5 \%$ of the total

15 body weight, this force approximated the weight of the arm for an average body mass of $67 \mathrm{~kg}$ (3.35

$16 \mathrm{~kg})(5,28)$. As each cane supported approximately the weight of half of the arm as the weight of the

17 arm was distributed across hand and shoulder, this preferred force offset the need to hold the arms

Formatted: Font color: Text 1

Formatted: Font color: Text 1

18 against gravity. To further probe into this effect, we estimated the weight of each participant's arm 
1 as the $5 \%$ of total body mass. Figure 2 shows these values against the sum of the forces applied on

2 both canes; this value was calculated as the average across time and divided by gravity. This led to

3 the same units as the $\mathrm{x}$ axis. The estimated arm weight correlated with the preferred force applied

4 on the canes as indicated by the significant Pearson correlation coefficient $(R=0.64, p<0.001)$. The

5 data cluster very closely around the identiy line, indicating that the preferred force is determined 6 by the weight of the participants arms.

8 Variability of Center of Pressure and Center of Mass. Figure 3 displays exemplary trials of Feet-CoP

9 (colored lines), Total-CoP (grey lines) and also of the center of mass CoM (black lines) for each 10 experimental condition (yellow shading represents the beam width). When standing on the ground 11 (Figure $3 \mathrm{~A}$ ), the fluctuations of $\mathrm{CoM}$ and CoP were considerably reduced compared to standing on 12 the beam without canes; this is especially pronounced in the AP direction (Figure 3B), which was 13 not surprising. When standing on the beam without canes, both CoP and CoM showed visibly larger 14 excursions, both in the AP and ML directions, again as expected.

15 The six panels in Figure $3 C$ show exemplary data from the same participant standing on the beam 16 with the canes on the ground. The excursions of both CoPs and CoM were significantly reduced 17 compared to those when standing on the beam without cane support and were similar to those 18 measured when standing on the ground (Figure $3 \mathrm{~A}, \mathrm{~B}$ ). The planar cane configuration led to visibly 19 smaller variability in the AP direction than the tripod configuration, especially in the maximum force 20 condition. In the minimum force condition, ML variability was similar in both Feet-CoP and Total$21 \mathrm{CoP}$, in both cane configurations. With increasing forces applied on the canes, the Feet-CoP 22 decreased its ML amplitude. In contrast, the Total-CoP went beyond the width of the beam, 23 indicating that the participants were moving their weight away from the feet and actively relying on 
1 the canes. Lastly, the fluctuations of the CoM, shown by the black lines, followed the changes of the

2 Total-CoP across different forces and cane placements and presented additional evidence that

3 participants shifted their weight beyond the base of support on the beam towards that provided by

4 the canes.

5 The CoP location along the beam changed between trials, even within the same participant. This

6 effect resulted from changing the distribution of body weight between the front and the back foot,

7 even without stepping off the beam between trials.

9 Comparison of Postural Sway On and Off the Beam. To first evaluate the difference between 10 balancing on the beam supported by canes with the two control conditions, the area of the CoM 11 served as a collective measure of balance performance. Figure 4A shows the CoM in the two control 12 conditions on and off the beam (white) next to the three force conditions on the beam (colored); 13 the data combined the two cane configurations to focus on the comparison with the two control 14 conditions. The figure makes it evident that standing on the beam without canes had the highest 15 degree of variability $(\beta=1682.1 \pm 290.2, p<0.001)$. More notable is that when on the beam with 16 canes the variability of the CoM declined to levels similar to the variability on the ground. 17 Additionally, when comparing to the different force conditions with pairwise post-hoc comparisons, 18 the two higher forces did not differ from standing on the ground (Pref: $p=0.08$, Max: $p=0.1$ ). Only 19 the minimum force condition showed a small but significant elevation compared to standing on the 20 ground (Min: $p=0.02$ ). Interestingly, when applying increasing force on the canes, the variability of 21 the CoM did not change $(p=1)$.

22 Figure $4 \mathrm{~B}$ shows participant averages of the $95 \%$ confidence interval of the CoP for the two control 23 conditions without cane support (white bars) next to the three force conditions (colored). To take 
1 into account the different nature of Total-CoP and Feet-CoP when participants used canes, the

2 results were separated. Again, the data were pooled for the two cane configurations to facilitate

3 comparison. As expected, standing on the beam without cane support significantly increased the

4 CoP excursions with respect to standing on the ground by a factor of $10(\beta=2953.6 \pm 236.2, p<$

5 0.001). However, when participants used the canes for support, the Total-CoP returned to values

6 similar to standing on the ground, as confirmed by the pairwise post-hoc comparisons $(p=1)$. The

7 Feet-CoP showed even smaller values than the Total-CoP with canes and the CoP on the ground

8 without canes $(p<0.001)$. While surprising at first, participants had three points of contact with the

9 floor that allowed them to rely less on foot-beam interaction and more on canes.

10 Taken together, these findings confirmed expectations that standing on the beam increased CoP 11 and CoM motions. However, both CoM and CoP variabilities approached the same level of variability 12 on the beam with canes and standing still on the ground. Given the instability when balancing on 13 the beam without canes, this gives first evidence of the significant mechanical effect of the canes.

Insert Figure 4 about here

15 Effect of Forces on Postural Balance in the ML Direction. This analysis focused on the conditions 16 when the participants stood on the beam and exerted three different force levels onto both canes.

17 The main metric is variability in the ML direction, computed as the standard deviation of the CoP 18 motion, as it is the most relevant direction for maintaining balance on a beam. Figure $5 \mathrm{~A}$ shows the $19 \mathrm{ML}$ variability of the Total-CoP against the average forces applied on the canes; the data points 20 represent all individual trials of all participants with force condition differentiated by color. Figure $215 \mathrm{~B}$ shows the same data averaged across the different force and arm conditions and pooled over all 22 participants. There was no evidence of any change with increasing force $(\beta=-0.000015 \pm 0.0001, p=$ 23 0.87). While different from what expected in Hypothesis 1 , this finding was consistent with previous 
1 results: ML variability was significantly attenuated with small forces at the support, and increasing

2 force levels did not further affect the Total-CoP $(10,11)$. However, the Total-CoP was affected by

3 the canes placement showing a slightly larger ML variability in the tripod condition

$4 \quad(\beta=0.0006 \pm 0.0002, p<0.001)$. The effect of arm configuration was counter to Hypothesis 3.

6 In contrast, the ML standard deviation of the Feet-CoP decreased with the average force for each 7 trial, as shown in Figure 5C. Figure 5D shows the pooled data of all participants for each 8 experimental condition. For both cane configurations the same trend was observed: applying a force 9 larger than minimum force reduced Feet-CoP variability $(\beta=-0.0008 \pm 0.00015, p<0.001)$. This 10 indicates that increasing force applied on the canes let participants rely less on foot-beam 11 interaction and more on canes. The variability in the ML direction for the Feet-CoP was not affected 12 by cane configuration $(\beta=-0.00016 \pm 0.0002, p=0.45)$, indicating that the spatial configuration of 13 the arms was not relevant for the ML direction of the Feet-CoP. This set of results on Feet-CoP is in 14 line with Hypothesis 1 and 3.

15 Effect of Forces on Postural Balance in the AP Direction. The standard deviations of both Feet-CoP 16 and Total-CoP were also compared in the AP direction, i.e., along the beam length. Figure 6 shows 17 that applying different levels of force did not affect AP variability neither in Feet-CoP $(\beta=$ $180.0003 \pm 0.0002, p=0.29)$ nor Total-CoP $(\beta=0.0002 \pm 0.0002, p=0.37)$. However, AP variability was 19 larger in the tripod condition than the planar condition, both for Feet-CoP and Total-CoP (Feet-CoP: $20 \beta=0.002 \pm 0.0007, p<0.01 ;$ Total-CoP: $\beta=0.002 \pm 0.0004, p<0.001)$. This confirmed that the tripod 21 cane condition allowed for more variability along the length of the beam and that participants 22 actually exploited this extended base of support by channeling noise in this task-irrelevant direction 23 (Hypothesis 3). 
2 Effect of Forces on Variability of Cane Motion. Even though participants were instructed to stand

3 still, some small movements in the body, arms, and hands were always present (7). This noise 4 inevitably transferred from the hand to the cane handle, deflecting the cane from the vertical 5 position. As a cane is an inverted pendulum, small lateral deflections destabilize the vertical cane.

6 To quantify these deflections, the path length of the hand on the cane handle was computed for 7 each trial. Figure 7A shows the paths traveled by the right and left hands of one participant over the 8 course of one trial in each force condition (marked by color). The path length of the hand increased 9 when more force was applied (right cane: $\beta=0.006 \pm 0.001, p<0.001$; left cane: $\beta=0.006 \pm 0.001, p$ $10<0.001)$. Figure 7B shows path lengths of all participants, plotted against the average force applied 11 on the respective cane; each point represents the path length traveled by the right or left hand 12 during one trial. It shows that the path length increased with the amount of force applied. Path 13 length in the minimum force condition was significantly different from the maximum force condition 14 for both hands (right hand: $p<0.001$, left hand: $p<0.001$ ). The preferred force condition was not 15 significantly different from the minimum condition for the right hand $(p=0.09)$ and only slightly 16 significant for the left hand $(p<0.05)$. These results suggest that higher forces indeed had a 17 destabilizing side-effects as expected (Hypothesis 2).

\section{Discussion}

20 Complementing much previous work on the role of light touch in postural balance, this study 21 detailed the mechanical effects of the canes on postural control in a challenging balance task. Are 22 these effects beneficial? Overall, while standing on a narrow beam, the use of canes improved 23 postural balance as evidenced in the reduced variability of the center of pressure (CoP) and the 
1 center of mass (CoM). These fluctuations declined to the same level as when standing on the floor.

2 This reduction in postural sway was observed for all force levels, even for relatively small forces

3 applied to the two canes. However, applying more force on the canes did not affect the medio-

4 lateral component of the Total-CoP, but only the Feet-CoP, i.e., measured directly under the feet on

5 the beam. The preferred force level on the canes corresponded to resting the arms' weight on the

6 canes, suggesting that participants exploited the mechanical support. However, when exerting

7 higher forces showed signs of destabilization, as the inverted pendulum of the canes was susceptible

8 to small excursions at the handle. In addition, having the canes in front rather than on the sides

9 allowed to channel variability in the antero-posterior direction, while the task-relevant, i.e., medio-

10 lateral direction remained relatively unaffected by this cane placement.

11 Perceptual Benefits of Canes. Numerous previous studies investigated the effect of light touch on 12 postural control and showed that increasing forces applied on a support surface did not provide 13 further benefit to reduce CoP motion $(9-12,15,27)$. However, some of the touch condition in 14 previous studies by Jeka and colleagues required subjects to apply a very small target force of $1 \mathrm{~N}$ 15 to $5 \mathrm{~N}$. Instructing participants to apply such a small force might create an additional goal beyond 16 maintaining balance: matching the touch force to the target level. Trying to accurately achieve the 17 target force may have introduced additional control processes in balance control. To avoid this 18 possibility, we did not provide participants with feedback of their applied force, but left them free 19 to choose the amount of force, as long as they chose three different levels. As the present study 20 also wanted to probe into the mechanical effects of canes on control, our study tested forces from $215 \mathrm{~N}$ to $100 \mathrm{~N}$ that extended well above the previously tested force conditions.

22 Our results on variability of the Total-CoP and the CoM showed again that, while canes were 23 generally helpful for balance, exerting the maximum level of force on the canes did not provide any 24 further stabilizing effect. In addition, when free to use their preferred force, participants did not 
1 choose a high level of force to maintain balance, presumably because there was no further benefit.

2 Rather, they stayed within a force level that reduced the need to hold their arms against gravity.

3 Taken together, these findings corroborated the widely accepted conclusion that even very light

4 touch provides perceptual information that enhances balance performance, similar to how visual

5 information stabilizes postural control (20). Hence, at first blush, these results seem to support the 6 conclusion that the mechanical effect of the support was negligible.

7 Mechanical Benefits of Canes on Postural Control. Intuitively, canes should facilitate balance, as

8 canes on the ground increase the base of support and that inherently changes the mechanics of the 9 system. But what are these mechanical effects and how do they affect demands on postural control? 10 Our data gave several indications that cane support went beyond being purely perceptual support 11 and also afforded mechanical benefits. First, as the additional contacts with the floor enlarged the 12 base of support, CoP and CoM went outside the beam width, which limits the base of support 13 without the canes. Therefore, humans indeed used the available larger base of support. Second, 14 while the extent of the fluctuations in CoP and CoM did not depend on the magnitude of forces 15 applied to the canes, they did depend on the placement of the canes. The standard deviations of 16 the Total-CoP and Feet-CoP in the tripod condition were significantly larger than in the planar 17 configuration. In particular, the tripod placement affected predominantly the antero-posterior 18 direction, channeling more variability in the task-irrelevant dimension. Third, the Feet-CoP motion 19 significantly decreased with increasing force on the canes, indicating that the more force applied on 20 the canes, the more control relied on their support to balance. Fourth, the preferred force 21 corresponded to $5 \%$ of average body weight, approximately equivalent to the weight of the 22 participant's arm (5). Hence, this choice of support reduced the effort required to hold one's arms, 23 while exploiting the new mechanical support provided by the additional devices. 
1 Mechanical Challenge due to Instability of the Canes. Applying forces on the vertical canes is an

2 isometric task with potential instability. Unlike in previous studies that tested forces applied on a

3 fixed surface, the canes were not inherently stable; rather, mechanically they presented an inverted

4 pendulum at its unstable equilibrium point. Hence, the inherent noise in the human sensorimotor

5 system introduces displacements. Assuming that noise increases with force, this destabilizing effect

6 increases with higher forces. On the other hand, as previously shown in the context of pushing a

7 stick against a wall, a downward compression force component on the cane increases the instability

8 of the inverted pendulum and any small excursion will destabilize the canes (22-24). Human joint

9 stiffness also increases with increasing force and may have counteracted this perturbing effect to

10 maintain postural balance (14). However, given the straight arms in our experiment, the stiffness at

11 the shoulder joint may have been limited as the increased displacements of the hands and canes

12 with higher forces showed. Interestingly, the variability of the CoM and the Total-CoP did not vary

13 with increasing force, indicating that the larger displacements at the hand may have been

14 compensated at the torso. Hence, these findings reveal that the cane support not only facilitated

15 balance, but also created complex control demands across the multi-segmented body.

16 Underlying Control Mechanisms. All together, these results present an intricate picture of how the 17 canes affected the control of postural stability, with some effects cancelling each other. What are 18 the potential control mechanisms underlying these observations? To begin, when standing on the 19 beam with canes, the variability of the CoM and Total-CoP in the ML or task-relevant direction were 20 essentially identical to those when standing on the ground. If the measured fluctuations when 21 standing on the ground are regarded as a floor effect determined by the noise level (as participants 22 were asked to stand as still as possible), then the use of canes enabled participants to minimize CoM 23 and Total-CoP variability to this lower bound. That could also be the reason why the different force 24 levels did not lead to further reductions. Second, when the canes formed a tripod, the fluctuations 
1 of the CoP were larger than in the planar condition, making use of the larger base of support.

2 Especially, the higher variability in the AP direction suggested that the controller did not constrain

3 fluctuations, i.e., allowing more variability in this task-irrelevant direction. Allowing variability in

4 directions orthogonal to what affects the task is usually interpreted as a reduction of control effort

$5(1,6)$. Third, control took advantage of the additional devices, evidenced in the preferred force level

6 that just off-set the weight of the arms while staying away from higher forces that potentially

7 introduced destabilizing effects. In summary, we speculate that the controller avoids high forces not

8 only because they require more effort without any obvious benefit, but also because they introduce

9 additional demands on neuro-muscular stiffness to counteract destabilizing forces. The controller

10 also allows fluctuations as long as the CoP stays within a certain region, whose limits are defined by

11 the margin of the base of support and by the noise of the system.

12 Limitations and Outlook. In the present study participants used canes to balance on a narrow beam 13 holding them with the arms extended. While this presented a clean geometric body configuration, 14 different mechanisms might be manifest if the canes were held with flexed arms or when walking 15 with one or two canes. Our metrics, ML and AP standard deviations and the total area of CoM and 16 CoP, could capture interesting features of the task, but they were scalar measures of data 17 distributions. Additional analyses could characterize the temporal evolution of the forces and their 18 relative centers of pressure. Further, recent work went beyond analyzing the point of application of 19 the force vector, and examined the orientation of the ground reaction force with respect to the 20 center of mass. This analysis revealed interesting information about the relative role of 21 biomechanics and control $(2,26)$. Applying these methods to the more challenging task of standing 22 on a narrow beam with canes could provide further information about the strategy adopted by the 23 controller when using canes. 
1 Postural balance improves with light touch on a stable surface suggesting perceptual benefits of

2 additional support. Here, new insights on the mechanical benefits of inherently unstable devices -

3 canes - are presented. Participants adapted to the novel mechanical system by trading off the

4 benefits from the additional support with the instability introduced by pushing on the canes.

5 Fluctuations of ground reaction forces show a channeling of noise in the task-irrelevant dimensions.

6 Such mechanical benefits provide a better understanding of the role of support when balance is

7 challenged, allowing future work to explore tailored rehabilitation protocols and the development

8 of novel assistive devices.

9

10 List of Abbreviations

11 CoP: Center of Pressure

12 CoM: Center of Mass

13 AP: Antero-Posterior

14 ML: Medio-Lateral

15

16 Declarations

17 Ethics approval and consent to participate.

18 The study was approved by the Institutional Review Board of Northeastern University.

19 Consent for publication

20 Not applicable.

21 Availability of data and materials 
1 The datasets used and/or analysed during the current study are available from the corresponding

2 author on reasonable request.

3 Competing interests

$4 \quad$ The authors declare that they have no competing interests.

\section{Fundings}

6 Jongwoo Lee was supported by a Samsung Scholarship. Neville Hogan was supported by NSF CRCNS-

7 1724135. Dagmar Sternad was supported by NSF CRCNS-1723998, and Marta Russo was supported 8 by NSF CRCNS-1723998 and the Italian Ministry of Health Starting Grant SG-2018-12366101.

\section{Authors contribution}

10 DS and NH designed the experimental protocol; JL collected the data; MR analyzed the data; MR

11 conducted the statistical analysis and created the figures; MR, JL, NH and DS discussed and

12 interpreted the results; MR wrote the first version of the manuscript; DS edited the first draft. All

13 authors jointly edited and approved the final version of the manuscript.

\section{Acknowledgements}

15 We would like to thank Christian Moses for his help in collecting the data. We would also like to 16 thank Dr. Randy Trumbower for lending us torque sensors.

17

18 References

19 1. Abe MO, Sternad D. Directionality in distribution and temporal structure of variability in skill 20 acquisition. Front Hum Neurosci 7: 225, 2013. doi: 10.3389/fnhum.2013.00225.

21 2. Boehm WL, Nichols KM, Gruben KG. Frequency-dependent contributions of sagittal-plane

foot force to upright human standing. J Biomech 83: 305-309, 2019. doi: 
10.1016/j.jbiomech.2018.11.039

2 3. Bryanton MA, Chodan SDC, Vander Meulen J, Fenrich KK, Misiaszek JE. The effect of light 3 touch on standing sway when the stability of the external touch reference becomes unreliable. Exp Brain Res 237: 663-672, 2019. doi: 10.1007/s00221-018-5455-4.

5 4. Chiovetto E, Huber ME, Sternad D, Giese MA. Low-dimensional organization of angular momentum during walking on a narrow beam. Sci Rep 8, 2018. doi: 10.1038/s41598-017$18142-y$.

8 5. De Leva P. Adjustments to zatsiorsky-seluyanov's segment inertia parameters. J Biomech 29: 1223-1230, 1996. doi: 10.1016/0021-9290(95)00178-6.

10 6. Dingwell JB, Cusumano JP. Re-interpreting detrended fluctuation analyses of stride-to-stride variability in human walking. Gait Posture 32: 348-353, 2010. doi: 10.1016/j.gaitpost.2010.06.004.

13 7. Faisal AA, Selen LPJ, Wolpert DM. Noise in the nervous system. Nat. Rev. Neurosci. 9: 292$303,2008$.

15 8. Hawke AL, Jensen RL. Are Trekking Poles Helping or Hindering Your Hiking Experience? A Review. Wilderness Environ Med 31: 482-488, 2020. doi: 10.1016/J.WEM.2020.06.009.

9. Jeka JJ. Light touch contact as a balance aid. Phys Ther 77: 476-487, 1997. doi: $10.1093 / \mathrm{ptj} / 77.5 .476$

10. Jeka JJ, Easton RD, Bentzen BL, Lackner JR. Haptic cues for orientation and postural control in sighted and blind individuals. Percept Psychophys 58: 409-423, 1996. doi: 10.3758/BF03206817. 
1 11. Jeka JJ, Lackner JR. Fingertip contact influences human postural control. Exp Brain Res 79: 495-502, 1994. doi: 10.1007/BF00229188.

3 12. Jeka JJ, Lackner JR. The role of haptic cues from rough and slippery surfaces in human postural control. Exp Brain Res 103: 267-276, 1995. doi: 10.1007/BF00231713.

13. Jones KE, Hamilton AF d. C, Wolpert DM. Sources of signal-dependent noise during isometric force production. J Neurophysiol 88: 1533-1544, 2002. doi: 10.1152/jn.2002.88.3.1533.

7 14. Lipps DB, Baillargeon EM, Ludvig D, Perreault EJ. Quantifying the multidimensional impedance of the shoulder during volitional contractions. Ann Biomed Eng 48: 2354-2369, 2020. doi: $10.1007 /$ s10439-020-02509-w.

15. Magre FL, Costa TDA da, Paiva AC de S, Moraes R, Mauerberg-deCastro E. Does the level of difficulty in balancing tasks affect haptic sensitivity via light touch? J Mot Behav 52: 1-12, 2020. doi: $10.1080 / 00222895.2019 .1565529$.

16. Misiaszek JE, Forero J, Hiob E, Urbanczyk T. Automatic postural responses following rapid displacement of a light touch contact during standing. Neuroscience 316: 1-12, 2016. doi: 10.1016/J.NEUROSCIENCE.2015.12.033.

17. Misiaszek JE, Vander Meulen J. Balance reactions to light touch displacements when standing on foam. Neurosci Lett 639: 13-17, 2017. doi: 10.1016/J.NEULET.2016.12.027.

18. Moraes R, Bedo BLS, Santos LO, Batistela RA, Santiago PRP, Mauerberg-deCastro E. Additional Haptic Information Provided by Anchors Reduces Postural Sway in Young Adults Less Than Does Light Touch. Front Neurosci 12: 346, 2018. doi: 10.3389/fnins.2018.00346.

19. Patla AE. Understanding the roles of vision in the control of human locomotion. Gait Posture 
$120 . \quad$ Peterka RJ. Sensorimotor integration in human postural control. J Neurophysiol 88: 10971118, 2002. doi: 10.1152/jn.2002.88.3.1097.

3 21. R Core Team. R: A language and environment for statistical computing [Online]. R Foundation for Statistical Computing: 2019. https://www.r-project.org/.

5 22. Rancourt D, Hogan N. Dynamics of pushing. J Mot Behav 33: 351-362, 2001. doi: 10.1080/00222890109601919.

7 23. Rancourt D, Hogan N. Stability in force-production tasks. J Mot Behav 33: 193-204, 2001. doi: 10.1080/00222890109603150.

9 24. Rancourt D, Hogan N. The biomechanics of force production. Adv Exp Med Biol 629: 645661, 2009. doi: 10.1007/978-0-387-77064-2_35.

25. Riley MA, Stoffregen TA, Grocki MJ, Turvey MT. Postural stabilization for the control of touching. Hum Mov Sci 18: 795-817, 1999. doi: 10.1016/S0167-9457(99)00041-X.

13 26. Shiozawa K, Lee J, Russo M, Sternad D, Hogan N. Frequency-dependent force direction elucidates neural control of balance. Res Sq PREPRINT, 2020. doi: 10.21203/RS.3.RS111213/V1.

27. Stramel DM, Carrera RM, Rahok SA, Stein J, Agrawal SK. Effects of a person-following lighttouch device during overground walking with visual perturbations in a virtual reality environment. IEEE Robot Autom Lett 4: 4139-4146, 2019. doi: 10.1109/Ira.2019.2931267.

19 28. Winter DA. Biomechanics and motor control of human movement: Fourth Edition. 2009. 
1 Fig 1: Experimental setup. Participants stood on a beam that was placed in a fixed position on a force plate, holding a cane in each hand. A set of 43 light-reflective markers measured displacements of the full body and the canes in 3D. The canes were instrumented with two $6 \mathrm{D}$ torque sensors at the bottom of each cane. The sketch shows the planar cane configuration where the two canes were placed to be on one line with the feet. In the tripod configuration, the canes were placed at a $45^{\circ}$ angle with the frontal plane to form a triangle with the feet.

9 Fig 2: Relation between estimated arm mass and force applied on the canes in the preferred force condition. Each data point represents the value for one trial ( 3 trials per participant). Arm mass has been estimated as $5 \%$ of total body mass of each participant. The force value on the $y$-axis was computed as the average force applied on one cane during one trial plus the average force applied on the other cane. Filled and empty points are trials in the Planar and

19 Fig 3: Representative paths of the center of pressure (CoP) and of the center of mass (CoM) in the significant correlation graphically shown by the blue solid line; the solid black line is the identity line. horizontal plane. The two CoPs and CoM for one trial for each of the different force instructions and the two postures are shown in a top-down view. A. Exemplary trial when standing on the ground. The grey line represents the CoP and the black line the CoM. B. CoP and CoM of one trial of the same participant are shown when standing on the beam without 
7 Fig 4: Variability metrics for the center of pressure (CoP) and center of mass (CoM) for all

canes. C. Each panel shows both the CoP at the feet (colored) and the total CoP (grey) for the three force conditions: minimum (green), preferred (blue), maximum (red); black lines show the center of mass (CoM). The two postures are identified by the drawings at the top of each panel. The beam is the light yellow area bounded by thin lines for visibility. For all conditions on and off the beam, the participant stood in tandem stance with the same foot in the front.

experimental conditions. The light yellow background indicates metrics for standing on the beam, while the white background on the left shows results for standing on the ground. The colored bars show the metrics when the participants used canes; green, blue and red differentiate the three force conditions. A: Area of the center of mass (CoM) quantified by the $95 \%$ tolerance ellipse. Each bar shows the mean and standard error $(n=16)$ for the different experimental conditions, pooled over all participants. The white bars on the left show the CoM area when participants stood on the ground and on the beam, without canes; the green, blue and red bars represent the three force conditions. B: Area of the center of pressure (CoP, Total-CoP and Feet-CoP) quantified by the $95 \%$ tolerance ellipse. Each bar shows the mean and standard error $(n=16)$ for the different experimental conditions, pooled over all participants. The two white bars show the CoP area when participants did not use canes. The lower value of CoP on the left represents the participants standing on the ground; the white bar shows the CoP area when participants stood on the beam. The colored bars show the Total-CoP and the Feet-CoP when the participants used canes. (significance levels: $* * *: p<0.001 ; *: p<0.05)$ 
1 Fig 5: Total center of pressure (Total-CoP) and center of pressure at the feet (Feet-CoP) in the medioTotal-CoP motion with respect to the sum of the forces applied on the canes; each data point is the average of one trial. Filled circles represent the planar posture, empty circles the tripod posture. B: Standard deviations of the ML-component of the Total-CoP motion for the two postures. Each data point represents one participant; different colors indicate different participants. C: ML-Feet-CoP against the sum of the forces applied on the canes for each trial. D: The ML component of the Feet-CoP for each force condition and for the two postures. Each data point represents one participant, different colors indicate different participants. (significance levels: $* * *: p<0.001 ; *: p<0.05$ )

Fig 6: Total center of pressure (Total-CoP) and center of pressure at the feet (Feet-CoP) in the Antero-Posterior (AP) direction for different cane conditions. A: Standard deviations of the AP-component of the Total-CoP motion for the two cane configurations. Data are pooled together within each force condition. Each data point represents one participant; different colors indicate different participants. B: The AP component of the Standar Deviation of the Feet-CoP for each force condition and for the two cane conditions. Each data point represents one participant, different colors indicate different participants. (*** indicates significance of $\mathrm{p}<0.001)$

Fig 7: Paths and path lengths of the left and right hands for different force instructions differentiated by color. A: Exemplary paths of the movements of the left and right hands from two point of view: $x-y$ at the top, $z-y$ below. Each colored line shows one trial in the three force conditions. 
Russo et al. Mechanical Effects of Canes on Postural Control 31 B: Path lengths for the left and right hands per trial are plotted against the average force applied to the cane. 


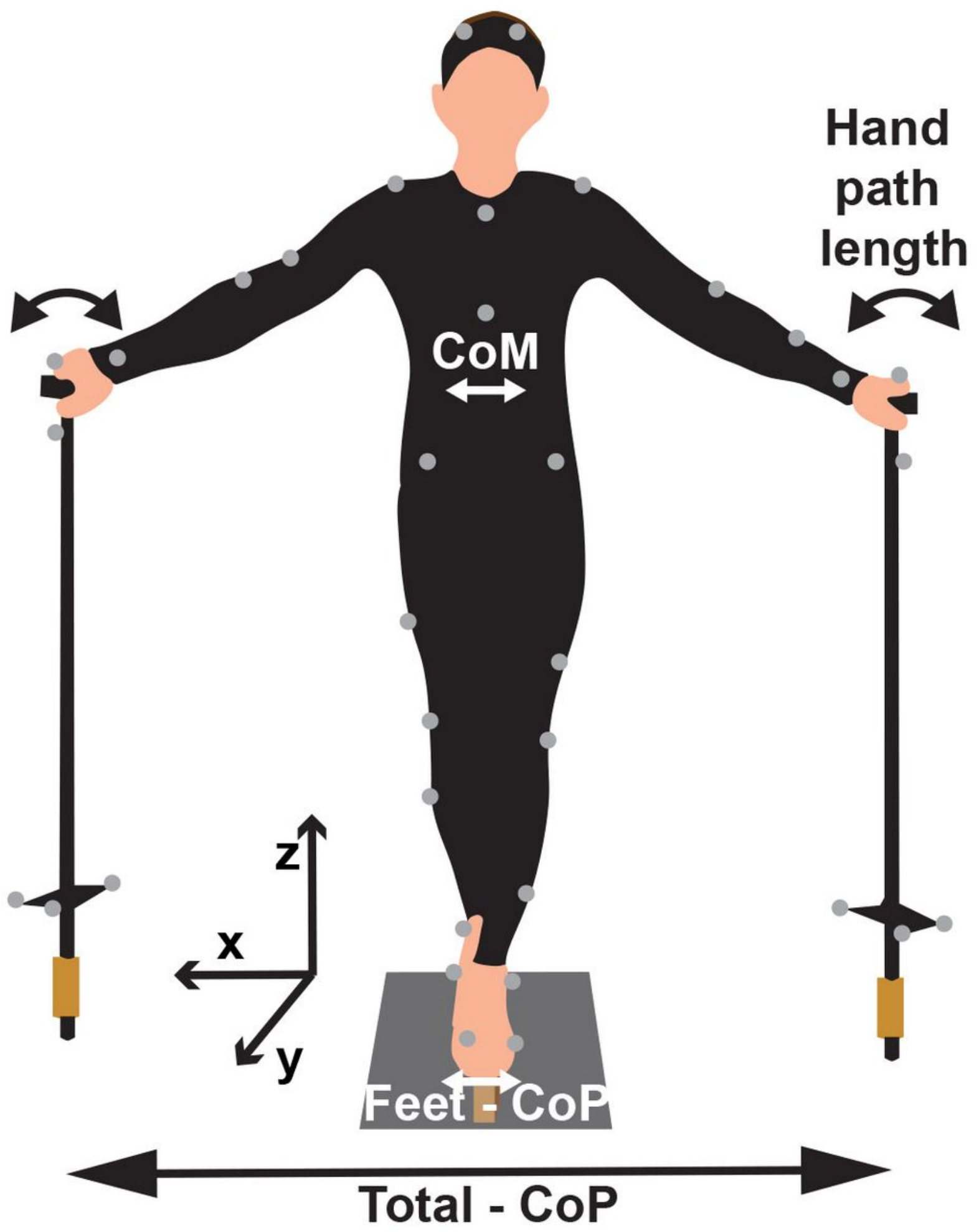

Figure 1

Experimental setup. Participants stood on a beam that was placed in a fixed position on a force plate, holding a cane in each hand. A set of 43 light-reflective markers measured displacements of the full body and the canes in 3D. The canes were instrumented with two 6D torque sensors at the bottom of each 
cane. The sketch shows the planar cane configuration where the two canes were placed to be on one line with the feet. In the tripod configuration, the canes were placed at a $45^{\circ}$ angle with the frontal plane to form a triangle with the feet.

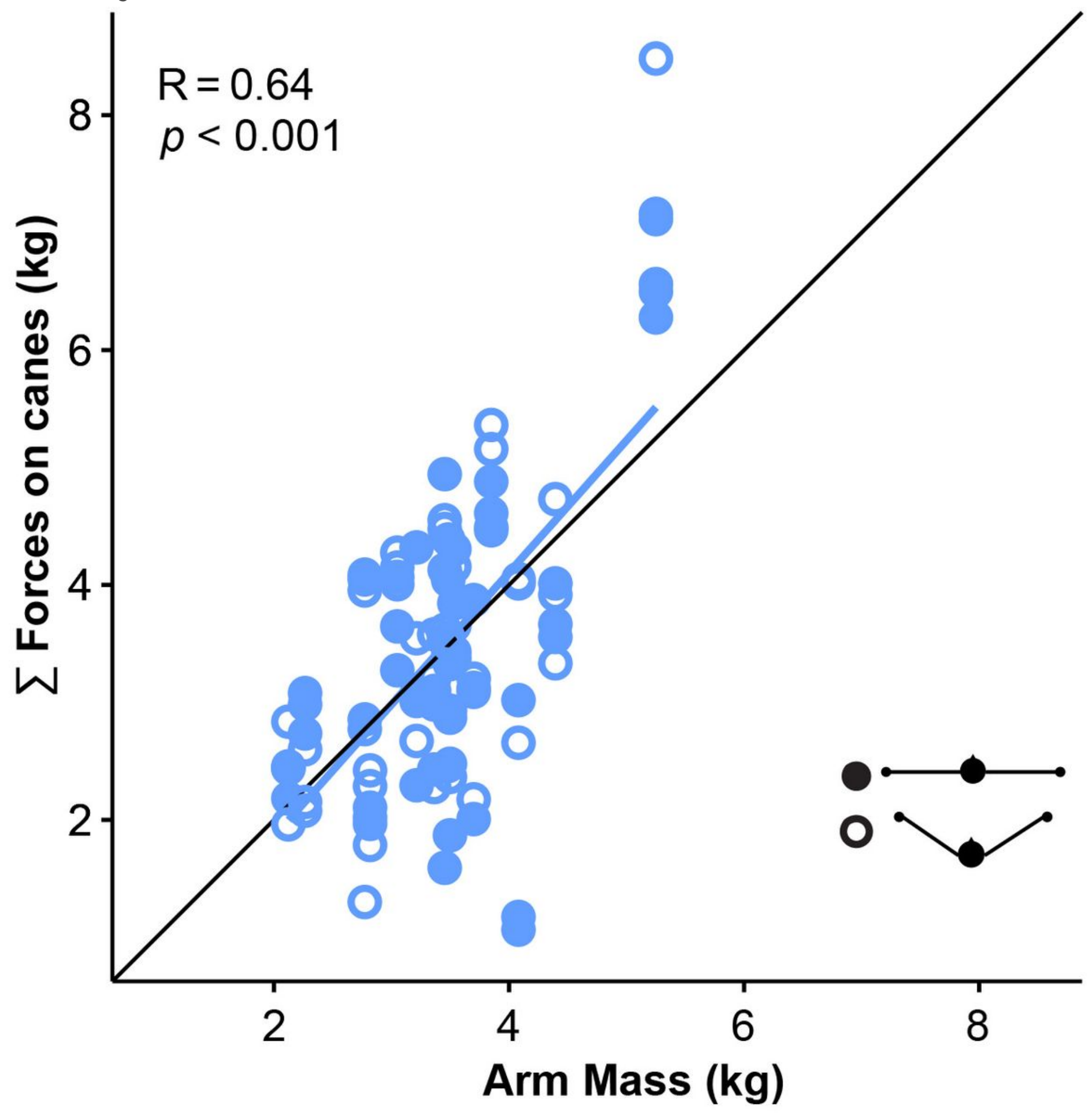

Figure 2

Relation between estimated arm mass and force applied on the canes in the preferred force condition. Each data point represents the value for one trial (3 trials per participant). Arm mass has been estimated as $5 \%$ of total body mass of each participant. The force value on the $y$-axis was computed as the average 
force applied on one cane during one trial plus the average force applied on the other cane. Filled and empty points are trials in the Planar and Tripod arm configurations, respectively. The Pearson correlation coefficient indicates a significant correlation graphically shown by the blue solid line; the solid black line is the identity line.

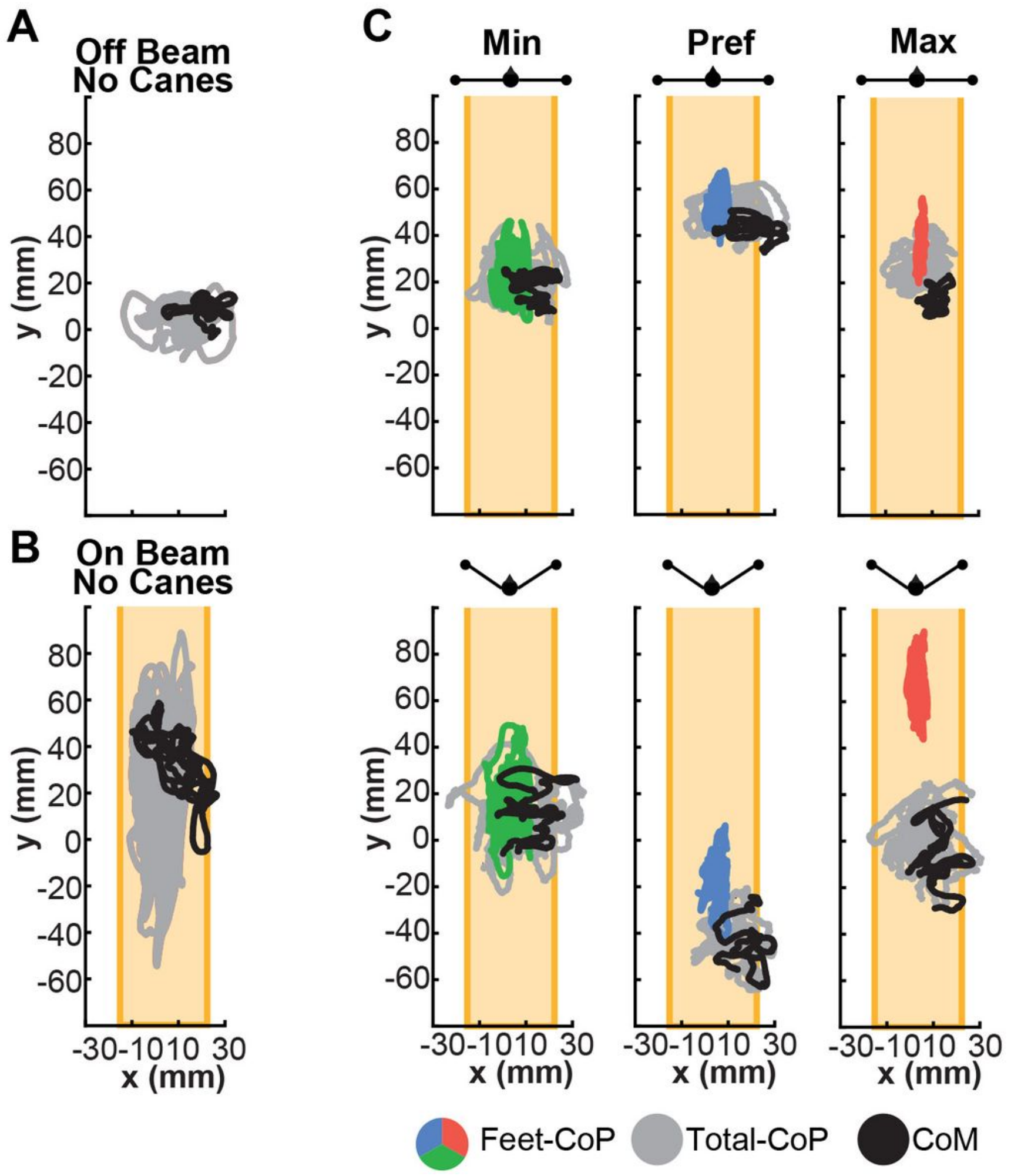

Figure 3 
Representative paths of the center of pressure (CoP) and of the center of mass (CoM) in the horizontal plane. The two CoPs and CoM for one trial for each of the different force instructions and the two postures are shown in a top-down view. A. Exemplary trial when standing on the ground. The grey line represents the CoP and the black line the CoM. B. CoP and CoM of one trial of the same participant are shown when standing on the beam without canes. C. Each panel shows both the CoP at the feet (colored) and the total CoP (grey) for the three force conditions: minimum (green), preferred (blue), maximum (red); black lines show the center of mass (CoM). The two postures are identified by the drawings at the top of each panel. The beam is the light yellow area bounded by thin lines for visibility. For all conditions on and off the beam, the participant stood in tandem stance with the same foot in the front. 

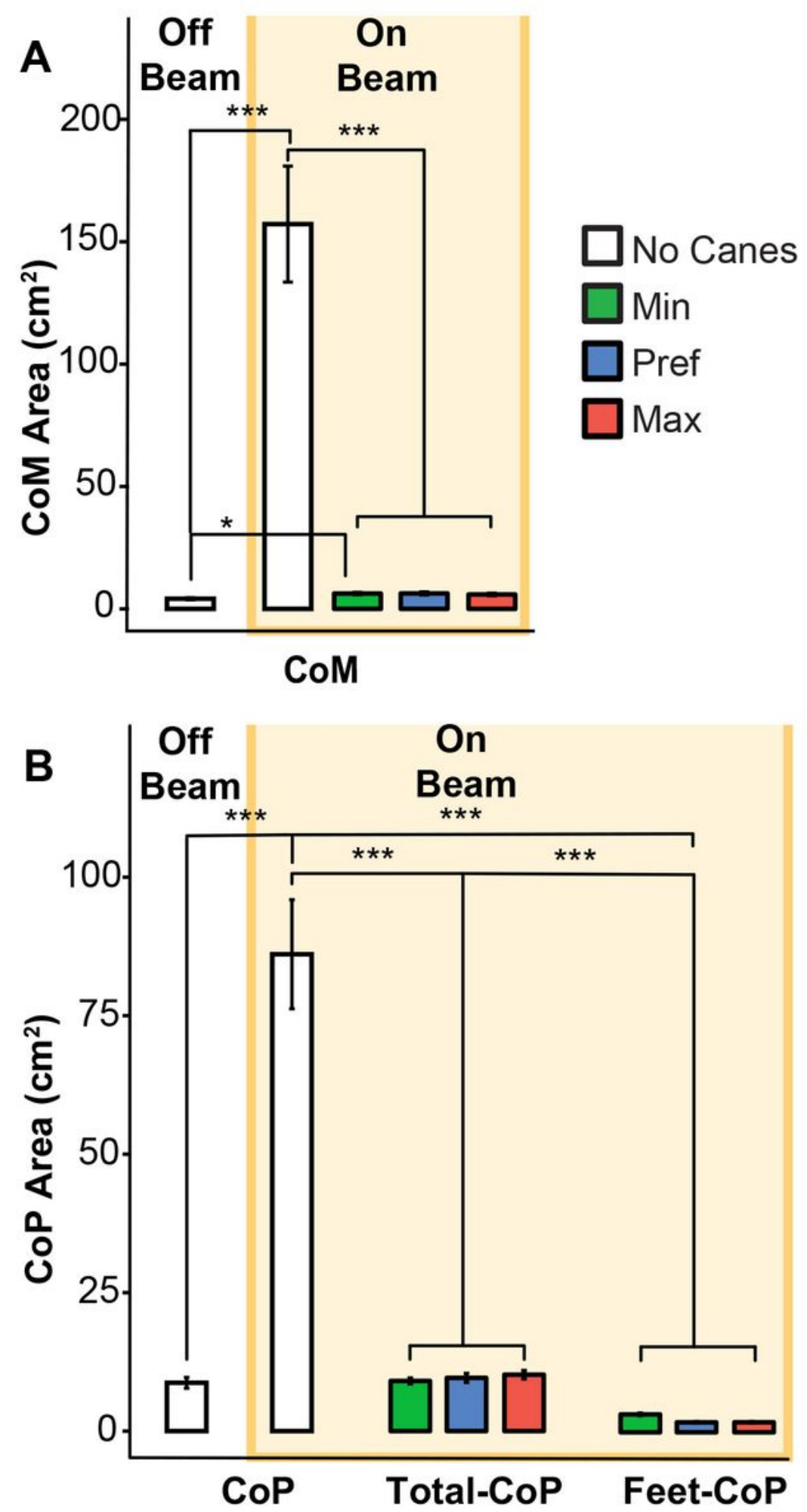

Figure 4

Variability metrics for the center of pressure (CoP) and center of mass (CoM) for all experimental conditions. The light yellow background indicates metrics for standing on the beam, while the white background on the left shows results for standing on the ground. The colored bars show the metrics when the participants used canes; green, blue and red differentiate the three force conditions. A: Area of the center of mass (CoM) quantified by the $95 \%$ tolerance ellipse. Each bar shows the mean and standard 
error $(n=16)$ for the different experimental conditions, pooled over all participants. The white bars on the left show the CoM area when participants stood on the ground and on the beam, without canes; the green, blue and red bars represent the three force conditions. B: Area of the center of pressure (CoP, TotalCoP and Feet-CoP) quantified by the $95 \%$ tolerance ellipse. Each bar shows the mean and standard error $(n=16)$ for the different experimental conditions, pooled over all participants. The two white bars show the CoP area when participants did not use canes. The lower value of CoP on the left represents the participants standing on the ground; the white bar shows the CoP area when participants stood on the beam. The colored bars show the Total-CoP and the Feet-CoP when the participants used canes. (significance levels: $* \star *: p<0.001 ; *: p<0.05$ )
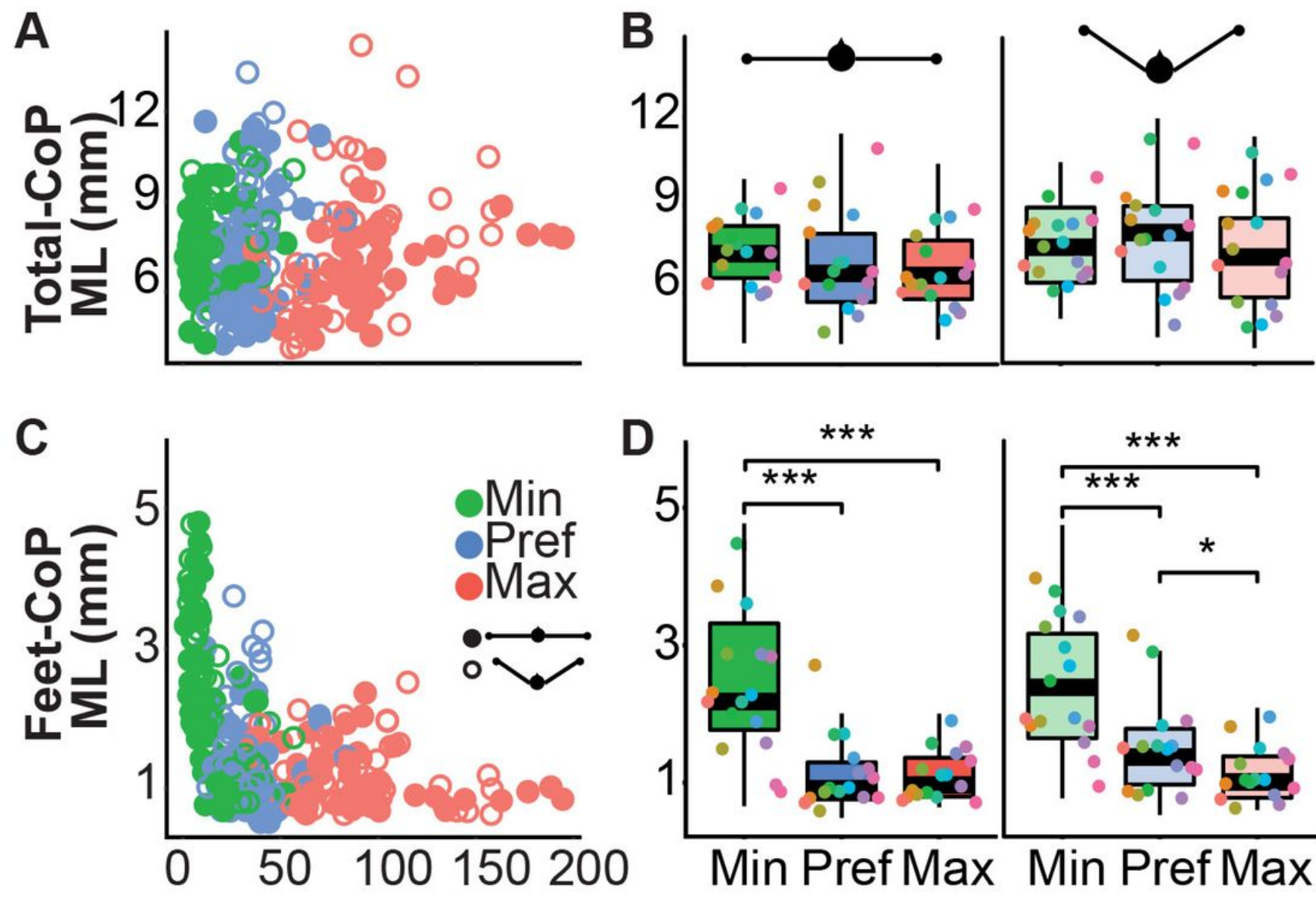
$\Sigma$ Forces on Canes (N) Force Condition

\section{Figure 5}

Total center of pressure (Total-CoP) and center of pressure at the feet (Feet-CoP) in the medio-lateral (ML) direction for different force conditions. A: Mediolateral (ML) component of the Total-CoP motion with respect to the sum of the forces applied on the canes; each data point is the average of one trial. Filled circles represent the planar posture, empty circles the tripod posture. B: Standard deviations of the MLcomponent of the Total-CoP motion for the two postures. Each data point represents one participant; different colors indicate different participants. C: ML-Feet-CoP against the sum of the forces applied on the canes for each trial. D: The ML component of the Feet-CoP for each force condition and for the two 
postures. Each data point represents one participant, different colors indicate different participants.

(significance levels: ***: $p<0.001 ; *$ : $p<0.05$ )
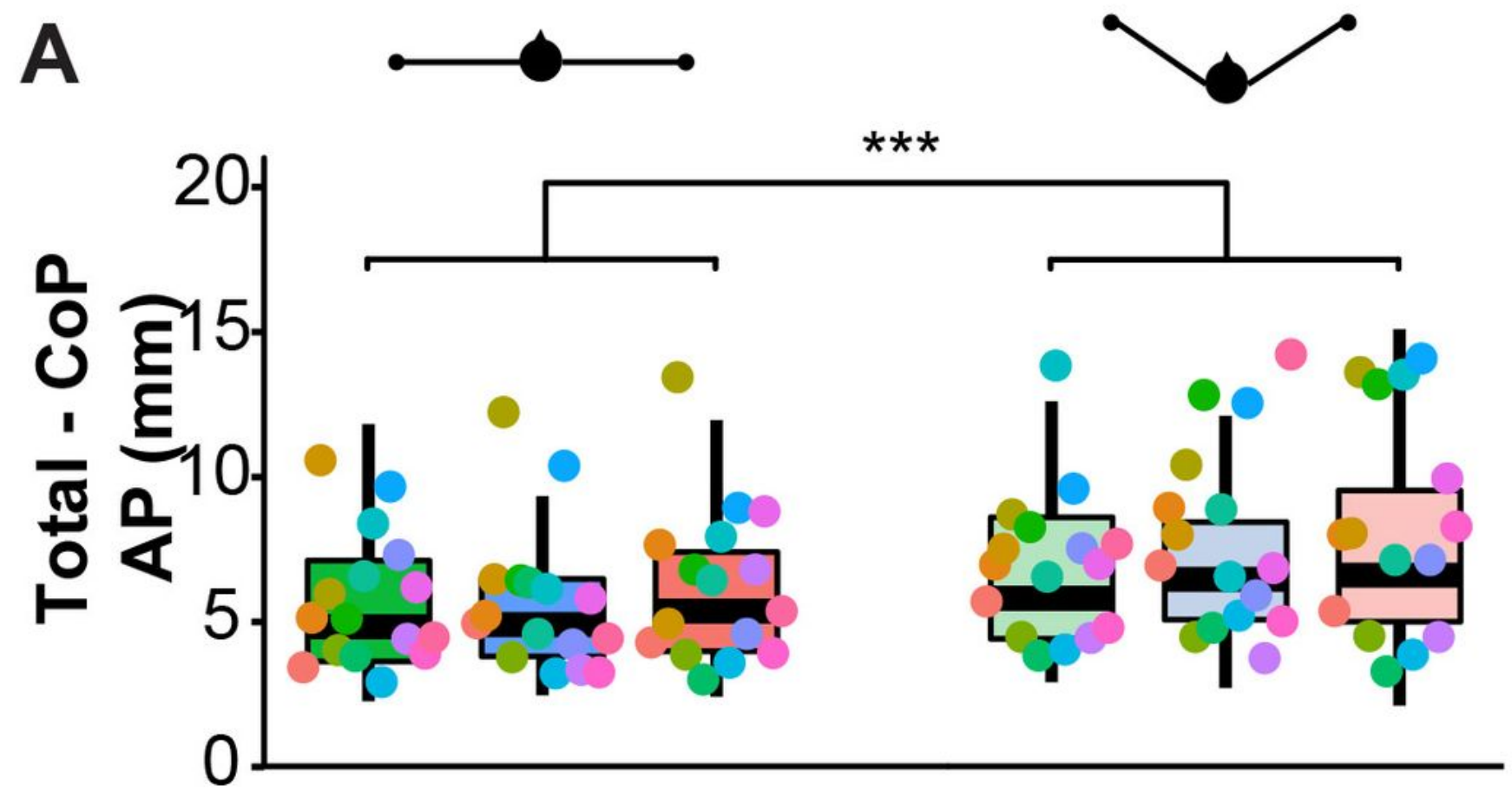

B

$\star * *$

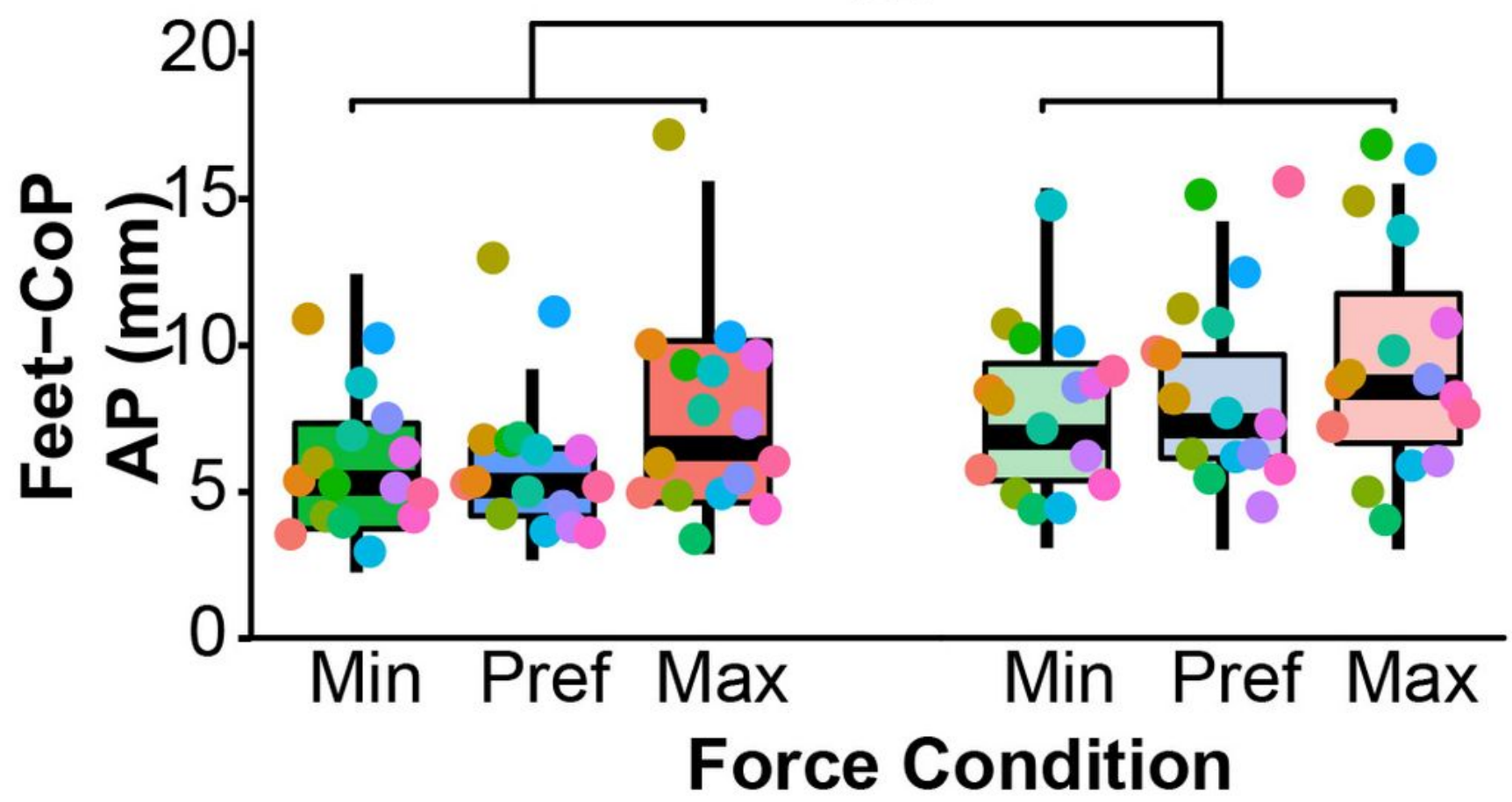

Figure 6

Total center of pressure (Total-CoP) and center of pressure at the feet (Feet-CoP) in the Antero-Posterior (AP) direction for different cane conditions. A: Standard deviations of the AP-component of the Total-CoP motion for the two cane configurations. Data are pooled together within each force condition. Each data 
point represents one participant; different colors indicate different participants. B: The AP component of the Standar Deviation of the Feet-CoP for each force condition and for the two cane conditions. Each data point represents one participant, different colors indicate different participants. ( ${ }^{\star \star \star}$ indicates significance of $p<0.001$ )
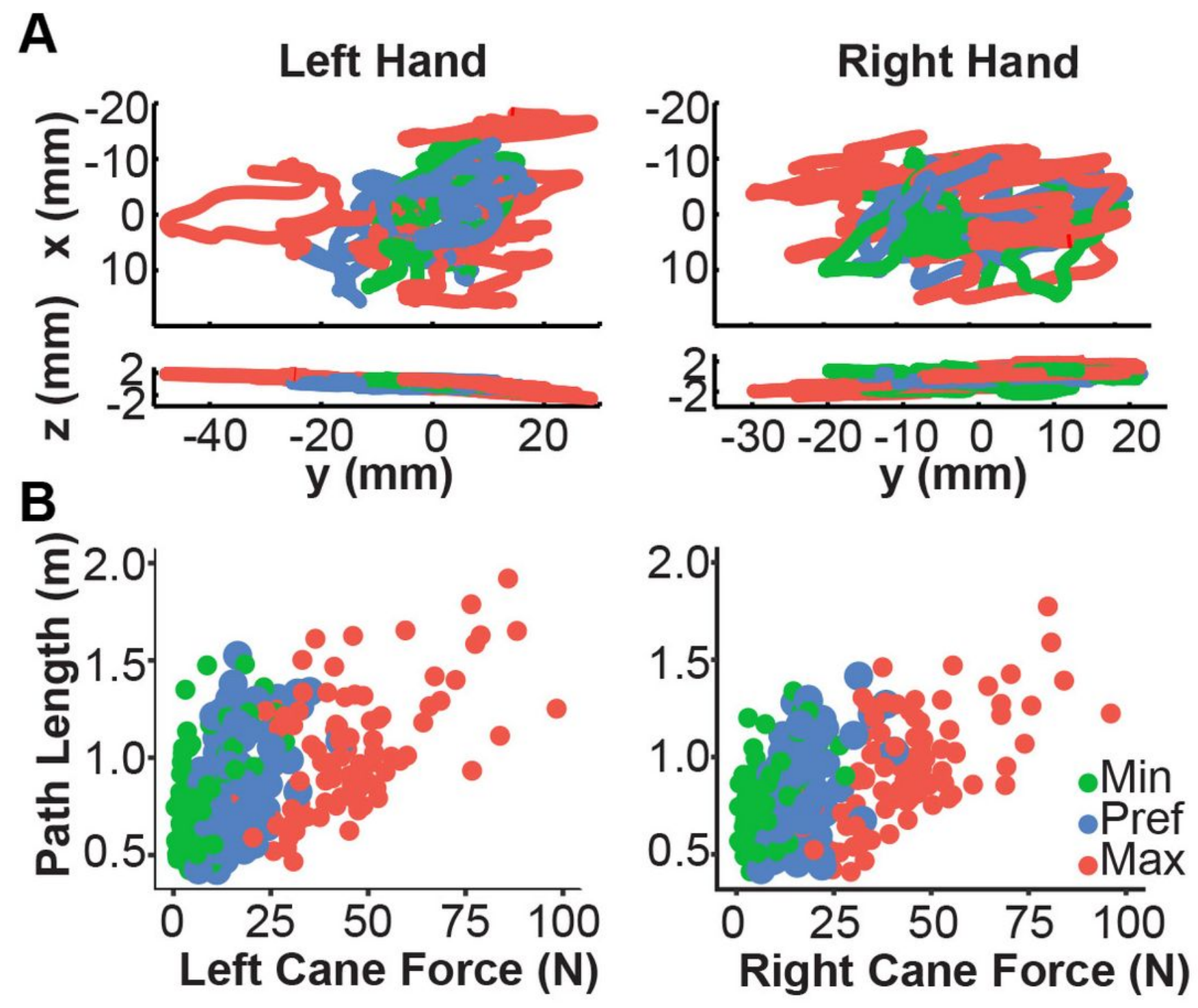

Figure 7

Paths and path lengths of the left and right hands for different force instructions differentiated by color. A: Exemplary paths of the movements of the left and right hands from two point of view: $x-y$ at the top, $z-$ $y$ below. Each colored line shows one trial in the three force conditions. B: Path lengths for the left and right hands per trial are plotted against the average force applied to the cane. 